NEUTRAL

FIRST ISOMER

Standard orientation:

\begin{tabular}{|c|c|c|c|c|c|}
\hline Center & Atomic & & itomic & Coordinat & \\
\hline Number & Numbe & & Type & $\mathrm{Y}$ & Z \\
\hline 1 & 8 & 0 & -0.008117 & 2.018030 & -0.072312 \\
\hline 2 & 6 & 0 & 0.157471 & 0.698543 & -0.062511 \\
\hline 3 & 7 & 0 & 1.506114 & 0.356416 & -0.015831 \\
\hline 4 & 13 & 0 & 1.877886 & 2.466612 & -0.016145 \\
\hline 5 & 6 & 0 & 1.874043 & -0.944538 & 0.022301 \\
\hline 6 & 7 & 0 & 1.036746 & -2.002335 & 0.036461 \\
\hline 7 & 6 & 0 & -0.258894 & -1.646462 & -0.027585 \\
\hline 8 & 7 & 0 & -1.339582 & -2.480959 & 0.008847 \\
\hline 9 & 6 & 0 & -2.537520 & -1.753261 & 0.070261 \\
\hline 10 & 7 & 0 & -2.164498 & -0.420563 & -0.102430 \\
\hline 11 & 6 & 0 & -0.776726 & -0.328981 & -0.085012 \\
\hline 12 & 1 & 0 & -2.769229 & 0.426104 & 0.000637 \\
\hline 13 & 1 & 0 & -3.452771 & -2.154171 & -0.368992 \\
\hline 14 & 1 & 0 & -1.271776 & -3.488836 & 0.114272 \\
\hline 15 & 7 & 0 & 3.221921 & -1.222365 & 0.008076 \\
\hline 16 & 1 & 0 & 3.470214 & -2.161529 & 0.306508 \\
\hline 17 & 1 & 0 & 3.847014 & -0.476511 & 0.300409 \\
\hline 18 & 7 & 0 & -3.090144 & 2.274706 & 0.132414 \\
\hline 19 & 1 & 0 & -3.576586 & 2.765302 & -0.623631 \\
\hline 20 & 1 & 0 & -3.446619 & 2.661057 & 1.011379 \\
\hline 21 & 1 & 0 & -2.091971 & 2.532280 & 0.070306 \\
\hline
\end{tabular}

ISOMER 2

Standard orientation:

\begin{tabular}{|c|c|c|c|c|c|}
\hline \multirow{2}{*}{$\begin{array}{l}\text { Center } \\
\text { Number }\end{array}$} & \multirow{2}{*}{$\begin{array}{l}\text { Atomic } \\
\text { Number }\end{array}$} & \multirow{2}{*}{\multicolumn{2}{|c|}{$\begin{array}{c}\text { Atomic } \\
\text { Type }\end{array}$}} & \multicolumn{2}{|c|}{ Coordinates (Angstroms } \\
\hline & & & & $Y$ & Z \\
\hline 1 & 8 & 0 & -2.401731 & -1.382053 & 0.014608 \\
\hline 2 & 6 & 0 & -1.267803 & -0.704120 & -0.017580 \\
\hline 3 & 7 & 0 & -1.481925 & 0.672747 & -0.010992 \\
\hline 4 & 13 & 0 & -3.583381 & 0.135046 & 0.037543 \\
\hline 5 & 6 & 0 & -0.433220 & 1.523409 & -0.024393 \\
\hline 6 & 7 & 0 & 0.869979 & 1.173939 & -0.020801 \\
\hline 7 & 6 & 0 & 1.057401 & -0.165612 & -0.037159 \\
\hline 8 & 7 & 0 & 2.254837 & -0.813040 & 0.002051 \\
\hline 9 & 6 & 0 & 2.069639 & -2.191124 & 0.109341 \\
\hline 10 & 7 & 0 & 0.684711 & -2.397495 & -0.071324 \\
\hline 11 & 6 & 0 & 0.043646 & -1.153058 & -0.044646 \\
\hline 12 & 1 & 0 & 0.224302 & -3.245871 & 0.246106 \\
\hline 13 & 1 & 0 & 2.797713 & -2.882726 & -0.319151 \\
\hline 14 & 1 & 0 & 3.155331 & -0.285353 & 0.034577 \\
\hline 15 & 7 & 0 & 4.099965 & 1.354804 & 0.041345 \\
\hline 16 & 1 & 0 & 4.645945 & 1.661572 & -0.769050 \\
\hline 17 & 1 & 0 & 4.601821 & 1.671256 & 0.876131 \\
\hline
\end{tabular}




$\begin{array}{rrrrrr}18 & 1 & 0 & 3.201163 & 1.858448 & 0.013821 \\ 19 & 7 & 0 & -0.718365 & 2.871093 & -0.085404 \\ 20 & 1 & 0 & 0.038823 & 3.485528 & 0.200494 \\ 21 & 1 & 0 & -1.649687 & 3.146664 & 0.214651\end{array}$

ISOMER 3

Standard orientation:

\begin{tabular}{|c|c|c|c|c|c|}
\hline \multirow{2}{*}{$\begin{array}{l}\text { Center } \\
\text { Number }\end{array}$} & \multirow{2}{*}{$\begin{array}{l}\text { Atomic } \\
\text { Number }\end{array}$} & \multirow{2}{*}{\multicolumn{2}{|c|}{$\begin{array}{l}\text { Atomic } \\
\text { Type }\end{array}$}} & \multicolumn{2}{|c|}{ Coordinates (Angstroms) } \\
\hline & & & & $X \quad Y$ & Z \\
\hline 1 & 8 & 0 & 2.477194 & -1.203612 & 0.048087 \\
\hline 2 & 6 & 0 & 1.268236 & -0.658723 & 0.016970 \\
\hline 3 & 7 & 0 & 1.343772 & 0.754072 & -0.013840 \\
\hline 4 & 13 & 0 & 3.574176 & 0.340550 & 0.025090 \\
\hline 5 & 6 & 0 & 0.253096 & 1.500537 & -0.033538 \\
\hline 6 & 7 & 0 & -1.007053 & 0.977665 & -0.070361 \\
\hline 7 & 6 & 0 & -1.142083 & -0.413891 & -0.050024 \\
\hline 8 & 7 & 0 & -2.297194 & -1.116077 & -0.056005 \\
\hline 9 & 6 & 0 & -1.915527 & -2.404324 & -0.020090 \\
\hline 10 & 7 & 0 & -0.527659 & -2.522036 & 0.007475 \\
\hline 11 & 6 & 0 & 0.001719 & -1.235929 & -0.007422 \\
\hline 12 & 1 & 0 & 0.009968 & -3.381447 & 0.040164 \\
\hline 13 & 1 & 0 & -2.579217 & -3.262149 & -0.017038 \\
\hline 14 & 1 & 0 & -3.800439 & 0.623938 & 0.063580 \\
\hline 15 & 7 & 0 & -3.759713 & 1.655547 & 0.100517 \\
\hline 16 & 1 & 0 & -4.212640 & 1.944544 & 0.972751 \\
\hline 17 & 1 & 0 & -4.341120 & 2.008473 & -0.665447 \\
\hline 18 & 1 & 0 & -1.887769 & 1.541846 & -0.001145 \\
\hline 19 & 7 & 0 & 0.363707 & 2.874795 & 0.036292 \\
\hline 20 & 1 & 0 & -0.375987 & 3.413649 & -0.409355 \\
\hline 21 & 1 & 0 & 1.301714 & 3.219115 & -0.158308 \\
\hline
\end{tabular}

ISOMER 4

Standard orientation:

\begin{tabular}{|c|c|c|c|c|c|}
\hline \multirow{2}{*}{$\begin{array}{l}\text { Center } \\
\text { Number }\end{array}$} & \multirow{2}{*}{$\begin{array}{l}\text { Atomic } \\
\text { Number }\end{array}$} & \multirow{2}{*}{\multicolumn{2}{|c|}{$\begin{array}{c}\text { Atomic } \\
\text { Type }\end{array}$}} & \multicolumn{2}{|c|}{ Coordinates (Angstroms) } \\
\hline & & & & $X \quad Y$ & Z \\
\hline 1 & 8 & 0 & -0.187740 & 1.990186 & -0.001370 \\
\hline 2 & 6 & 0 & 0.093272 & 0.679900 & -0.000603 \\
\hline 3 & 7 & 0 & 1.477598 & 0.445460 & -0.014573 \\
\hline 4 & 13 & 0 & 1.512967 & 2.790896 & -0.030941 \\
\hline 5 & 6 & 0 & 1.973382 & -0.771027 & 0.008770 \\
\hline 6 & 7 & 0 & 1.187857 & -1.906695 & -0.047533 \\
\hline 7 & 6 & 0 & -0.210432 & -1.730680 & -0.026782 \\
\hline 8 & 7 & 0 & -1.154862 & -2.694195 & -0.021704 \\
\hline 9 & 6 & 0 & -2.314477 & -2.013504 & -0.003244 \\
\hline 10 & 7 & 0 & -2.120823 & -0.644935 & -0.001468 \\
\hline 11 & 6 & 0 & -0.753471 & -0.434599 & -0.012268 \\
\hline 12 & 1 & 0 & -2.812670 & 0.137319 & 0.014326 \\
\hline
\end{tabular}




$\begin{array}{rrrrrr}13 & 1 & 0 & -3.301524 & -2.465018 & 0.006711 \\ 14 & 1 & 0 & 1.570201 & -2.791107 & 0.285514 \\ 15 & 7 & 0 & 3.338334 & -0.979902 & 0.132249 \\ 16 & 1 & 0 & 3.716608 & -1.736663 & -0.438921 \\ 17 & 1 & 0 & 3.872860 & -0.117292 & 0.037021 \\ 18 & 7 & 0 & -3.309642 & 1.970432 & 0.037580 \\ 19 & 1 & 0 & -3.755734 & 2.367856 & 0.869486 \\ 20 & 1 & 0 & -2.334439 & 2.308679 & 0.025614 \\ 21 & 1 & 0 & -3.780825 & 2.381398 & -0.773648\end{array}$

ISOMER 5

Standard orientation:

\begin{tabular}{|c|c|c|c|c|c|}
\hline \multirow{2}{*}{$\begin{array}{l}\text { Center } \\
\text { Number }\end{array}$} & \multirow{2}{*}{\multicolumn{2}{|c|}{$\begin{array}{l}\text { Atomic } \\
\text { Number }\end{array}$}} & & \multicolumn{2}{|c|}{ Coordinates (Angstroms) } \\
\hline & & & & $\mathrm{Y}$ & Z \\
\hline 1 & 8 & 0 & 0.238370 & 1.938884 & -0.047671 \\
\hline 2 & 6 & 0 & 0.311342 & 0.627100 & -0.037102 \\
\hline 3 & 7 & 0 & 1.492201 & -0.141640 & -0.043582 \\
\hline 4 & 6 & 0 & 1.435143 & -1.516388 & -0.020061 \\
\hline 5 & 7 & 0 & 0.325379 & -2.257082 & -0.007032 \\
\hline 6 & 6 & 0 & -0.818822 & -1.508389 & 0.011770 \\
\hline 7 & 7 & 0 & -2.141292 & -1.868750 & 0.040037 \\
\hline 8 & 6 & 0 & -2.928064 & -0.709035 & 0.038457 \\
\hline 9 & 7 & 0 & -2.124749 & 0.401413 & 0.007632 \\
\hline 10 & 13 & 0 & -1.687285 & 2.537859 & -0.022161 \\
\hline 11 & 6 & 0 & -0.845854 & -0.121169 & -0.008407 \\
\hline 12 & 1 & 0 & -4.010320 & -0.728636 & 0.053246 \\
\hline 13 & 1 & 0 & -2.498393 & -2.820501 & 0.063025 \\
\hline 14 & 7 & 0 & 2.651483 & -2.175581 & 0.049856 \\
\hline 15 & 1 & 0 & 2.568804 & -3.167014 & -0.167041 \\
\hline 16 & 1 & 0 & 3.443578 & -1.719284 & -0.398153 \\
\hline 17 & 1 & 0 & 2.392790 & 0.392792 & 0.005232 \\
\hline 18 & 7 & 0 & 3.363729 & 2.005705 & 0.081208 \\
\hline 19 & 1 & 0 & 3.843403 & 2.278971 & 0.944316 \\
\hline 20 & 1 & 0 & 3.929251 & 2.361378 & -0.695476 \\
\hline 21 & 1 & 0 & 2.468893 & 2.517883 & 0.059542 \\
\hline
\end{tabular}

ISOMER 6

Standard orientation:

\begin{tabular}{|c|c|c|c|c|c|}
\hline \multirow{2}{*}{$\begin{array}{l}\text { Center } \\
\text { Number }\end{array}$} & \multirow{2}{*}{$\begin{array}{l}\text { Atomic } \\
\text { Number }\end{array}$} & \multirow{2}{*}{\multicolumn{2}{|c|}{$\begin{array}{l}\text { Atomic } \\
\text { Type }\end{array}$}} & \multicolumn{2}{|c|}{ Coordinates (Angstroms) } \\
\hline & & & & $\begin{array}{ll}X & Y\end{array}$ & Z \\
\hline 1 & 8 & 0 & -1.626735 & -0.445523 & -0.001119 \\
\hline 2 & 6 & 0 & -0.370158 & 0.015699 & -0.024304 \\
\hline 3 & 7 & 0 & -0.179709 & 1.364138 & -0.012050 \\
\hline 4 & 13 & 0 & -3.014956 & 0.794711 & 0.054432 \\
\hline 5 & 7 & 0 & -4.184679 & -1.210839 & -0.030288 \\
\hline 6 & 1 & 0 & -4.646657 & -1.453609 & 0.853545 \\
\hline 7 & 1 & 0 & -4.898234 & -1.259632 & -0.765615 \\
\hline 8 & 1 & 0 & -3.468607 & -1.922448 & -0.221762 \\
\hline
\end{tabular}




$\begin{array}{cccccc}9 & 6 & 0 & 1.078879 & 1.837642 & -0.016199 \\ 10 & 7 & 0 & 2.233524 & 1.118989 & -0.002623 \\ 11 & 6 & 0 & 2.008605 & -0.197446 & -0.024762 \\ 12 & 7 & 0 & 2.947021 & -1.199490 & 0.003732 \\ 13 & 6 & 0 & 2.331342 & -2.464604 & 0.147410 \\ 14 & 7 & 0 & 0.950528 & -2.208120 & -0.074975 \\ 15 & 6 & 0 & 0.744461 & -0.821236 & -0.045852 \\ 16 & 1 & 0 & 0.252016 & -2.840421 & 0.307552 \\ 17 & 1 & 0 & 2.791679 & -3.322766 & -0.356349 \\ 18 & 1 & 0 & 3.937687 & -1.031600 & 0.148179 \\ 19 & 7 & 0 & 1.225198 & 3.212427 & -0.076387 \\ 20 & 1 & 0 & 2.126203 & 3.559458 & 0.242158 \\ 21 & 1 & 0 & 0.412269 & 3.743890 & 0.224002 \\ ---------------------------------------------------------\end{array}$

ISOMER 7

Standard orientation:

\begin{tabular}{|c|c|c|c|c|c|}
\hline \multirow{2}{*}{$\begin{array}{l}\text { Center } \\
\text { Number }\end{array}$} & \multirow{2}{*}{\multicolumn{2}{|c|}{$\begin{array}{l}\text { Atomic } \\
\text { Number }\end{array}$}} & \multirow{2}{*}{$\begin{array}{l}\text { Atomic } \\
\text { Type }\end{array}$} & \multicolumn{2}{|c|}{ Coordinates (Angstroms) } \\
\hline & & & & $X$ & Z \\
\hline 1 & 8 & 0 & 2.770483 & 0.051335 & 0.018301 \\
\hline 2 & 6 & 0 & 1.542871 & 0.511706 & -0.008116 \\
\hline 3 & 7 & 0 & 1.156060 & 1.883593 & 0.030825 \\
\hline 4 & 6 & 0 & -0.191598 & 2.232543 & 0.003812 \\
\hline 5 & 7 & 0 & -1.216795 & 1.400562 & 0.016738 \\
\hline 6 & 7 & 0 & -4.529467 & -1.197405 & 0.021094 \\
\hline 7 & 1 & 0 & -4.899189 & -0.637221 & -0.753694 \\
\hline 8 & 1 & 0 & -4.972602 & -2.118942 & -0.047649 \\
\hline 9 & 1 & 0 & -4.880729 & -0.767557 & 0.882802 \\
\hline 10 & 6 & 0 & -0.847738 & 0.072826 & -0.019347 \\
\hline 11 & 7 & 0 & -1.590148 & -1.078975 & -0.013156 \\
\hline 12 & 6 & 0 & -0.716052 & -2.154951 & -0.015533 \\
\hline 13 & 7 & 0 & 0.585268 & -1.733284 & -0.020706 \\
\hline 14 & 13 & 0 & 2.796413 & -1.929777 & 0.017919 \\
\hline 15 & 6 & 0 & 0.473748 & -0.355398 & -0.025920 \\
\hline 16 & 1 & 0 & -1.052016 & -3.185250 & -0.010608 \\
\hline 17 & 1 & 0 & -2.633919 & -1.146598 & -0.003564 \\
\hline 18 & 7 & 0 & -0.437265 & 3.599127 & -0.083950 \\
\hline 19 & 1 & 0 & -1.426134 & 3.805084 & 0.053669 \\
\hline 20 & 1 & 0 & 0.165461 & 4.188668 & 0.490517 \\
\hline 21 & 1 & 0 & 1.840946 & 2.582550 & -0.256108 \\
\hline
\end{tabular}

CATIONS

CATION I'

Standard orientation:

$\begin{array}{llll}\text { Center Atomic Atomic } & \text { Coordinates (Angstroms) } \\ \text { Number Number Type } & \text { X Y Y Z }\end{array}$

$\begin{array}{llllll}1 & 8 & 0 & 0.046600 & 1.983625 & -0.001257\end{array}$ 


$\begin{array}{cccccc}2 & 6 & 0 & 0.277003 & 0.698775 & -0.000427 \\ 3 & 7 & 0 & 1.575374 & 0.315179 & 0.000541 \\ 4 & 13 & 0 & 1.866221 & 2.719643 & -0.001179 \\ 5 & 6 & 0 & 1.914342 & -1.011471 & 0.000898 \\ 6 & 7 & 0 & 1.042305 & -2.051611 & 0.000498 \\ 7 & 6 & 0 & -0.220367 & -1.646352 & -0.000089 \\ 8 & 7 & 0 & -1.365189 & -2.443376 & -0.000675 \\ 9 & 6 & 0 & -2.460479 & -1.635758 & -0.001394 \\ 10 & 7 & 0 & -2.087482 & -0.359175 & -0.001425 \\ 11 & 6 & 0 & -0.698194 & -0.325290 & -0.000476 \\ 12 & 1 & 0 & -2.732781 & 0.566353 & -0.000098 \\ 13 & 1 & 0 & -3.485770 & -1.993670 & -0.001750 \\ 14 & 1 & 0 & -1.376303 & -3.462622 & -0.000621 \\ 15 & 7 & 0 & 3.229927 & -1.309913 & 0.001741 \\ 16 & 1 & 0 & 3.517092 & -2.283149 & 0.001604 \\ 17 & 1 & 0 & 3.928612 & -0.575583 & 0.001490 \\ 18 & 7 & 0 & -3.378383 & 1.988685 & 0.002713 \\ 19 & 1 & 0 & -3.947315 & 2.215811 & -0.820089 \\ 20 & 1 & 0 & -3.946265 & 2.213433 & 0.826890 \\ 21 & 1 & 0 & -2.580648 & 2.637108 & 0.003127 \\ -------------------------------------------------------------\end{array}$

\section{CATION II'}

Standard orientation:

\begin{tabular}{|c|c|c|c|c|c|}
\hline \multirow{2}{*}{$\begin{array}{l}\text { Center } \\
\text { Number }\end{array}$} & \multirow{2}{*}{$\begin{array}{l}\text { Atomic } \\
\text { Number }\end{array}$} & \multirow{2}{*}{\multicolumn{2}{|c|}{$\begin{array}{l}\text { Atomic } \\
\text { Type }\end{array}$}} & \multicolumn{2}{|c|}{ Coordinates (Angstroms) } \\
\hline & & & & $X \quad Y$ & Z \\
\hline 1 & 8 & 0 & 2.344861 & -1.298574 & -0.000469 \\
\hline 2 & 6 & 0 & 1.312233 & -0.491264 & -0.000533 \\
\hline 3 & 7 & 0 & 1.520218 & 0.836318 & -0.000495 \\
\hline 4 & 13 & 0 & 3.986589 & -0.353113 & 0.001252 \\
\hline 5 & 6 & 0 & 0.456856 & 1.697993 & -0.000394 \\
\hline 6 & 7 & 0 & -0.858735 & 1.359703 & -0.000405 \\
\hline 7 & 6 & 0 & -1.037961 & 0.042432 & -0.000386 \\
\hline 8 & 7 & 0 & -2.256702 & -0.639974 & -0.000166 \\
\hline 9 & 6 & 0 & -1.998518 & -1.958777 & -0.000098 \\
\hline 10 & 7 & 0 & -0.671079 & -2.169515 & -0.000369 \\
\hline 11 & 6 & 0 & -0.030966 & -0.935588 & -0.000536 \\
\hline 12 & 1 & 0 & -0.217400 & -3.081870 & -0.000081 \\
\hline 13 & 1 & 0 & -2.747276 & -2.745200 & 0.000078 \\
\hline 14 & 1 & 0 & -3.255613 & -0.166074 & -0.000209 \\
\hline 15 & 7 & 0 & -4.673714 & 0.633775 & 0.001072 \\
\hline 16 & 1 & 0 & -5.262247 & 0.466222 & 0.824813 \\
\hline 17 & 1 & 0 & -5.266106 & 0.462523 & -0.819136 \\
\hline 18 & 1 & 0 & -4.461732 & 1.638396 & -0.001675 \\
\hline 19 & 7 & 0 & 0.753387 & 3.016304 & 0.000227 \\
\hline 20 & 1 & 0 & 0.002670 & 3.697865 & -0.001718 \\
\hline 21 & 1 & 0 & 1.719673 & 3.322140 & -0.001963 \\
\hline
\end{tabular}

CATION III'

Standard orientation: 


\begin{tabular}{|c|c|c|c|c|c|}
\hline Center & Atomic & & omic & Coordinates & (Angstroms) \\
\hline Number & Numbe & & Type & $\mathrm{Y}$ & Z \\
\hline 1 & 8 & 0 & 2.550339 & 0.526002 & 0.003007 \\
\hline 2 & 6 & 0 & 1.289907 & 0.166139 & -0.000093 \\
\hline 3 & 7 & 0 & 0.972989 & -1.146871 & -0.002755 \\
\hline 4 & 13 & 0 & 4.026236 & -0.600801 & 0.004325 \\
\hline 5 & 6 & 0 & -0.313535 & -1.539007 & -0.004523 \\
\hline 6 & 7 & 0 & -1.372423 & -0.675491 & -0.005991 \\
\hline 7 & 6 & 0 & -1.087276 & 0.661658 & -0.004179 \\
\hline 8 & 7 & 0 & -1.979185 & 1.690548 & -0.004294 \\
\hline 9 & 6 & 0 & -1.204395 & 2.778674 & -0.001421 \\
\hline 10 & 7 & 0 & 0.131005 & 2.490758 & 0.000445 \\
\hline 11 & 6 & 0 & 0.237704 & 1.108460 & -0.001193 \\
\hline 12 & 1 & 0 & 0.899614 & 3.157980 & 0.002691 \\
\hline 13 & 1 & 0 & -1.575618 & 3.801276 & -0.000787 \\
\hline 14 & 1 & 0 & -4.390996 & -0.140278 & 0.022531 \\
\hline 15 & 7 & 0 & -4.101306 & -1.126409 & 0.010449 \\
\hline 16 & 1 & 0 & -4.548739 & -1.545347 & -0.812356 \\
\hline 17 & 1 & 0 & -4.535721 & -1.562110 & 0.831527 \\
\hline 18 & 1 & 0 & -2.418030 & -0.980680 & -0.000669 \\
\hline 19 & 7 & 0 & -0.567746 & -2.866733 & 0.003152 \\
\hline 20 & 1 & 0 & -1.510397 & -3.236944 & -0.038172 \\
\hline 21 & 1 & 0 & 0.218343 & -3.507656 & -0.023644 \\
\hline
\end{tabular}

CATION V

Standard orientation:

\begin{tabular}{|c|c|c|c|c|c|}
\hline \multirow{2}{*}{$\begin{array}{l}\text { Center } \\
\text { Number }\end{array}$} & \multirow{2}{*}{$\begin{array}{l}\text { Atomic } \\
\text { Number }\end{array}$} & \multirow{2}{*}{\multicolumn{2}{|c|}{$\begin{array}{l}\text { Atomic } \\
\text { Type }\end{array}$}} & \multicolumn{2}{|c|}{ Coordinates (Angstroms) } \\
\hline & & & & $x$ & Z \\
\hline 1 & 8 & 0 & -0.119528 & 1.962349 & -0.002405 \\
\hline 2 & 6 & 0 & -0.171632 & 0.667520 & -0.002046 \\
\hline 3 & 7 & 0 & -1.362980 & -0.015178 & -0.001547 \\
\hline 4 & 6 & 0 & -1.412115 & -1.407753 & -0.000321 \\
\hline 5 & 7 & 0 & -0.340790 & -2.205566 & 0.000454 \\
\hline 6 & 6 & 0 & 0.818302 & -1.531562 & 0.000069 \\
\hline 7 & 7 & 0 & 2.117585 & -1.998496 & 0.001328 \\
\hline 8 & 6 & 0 & 2.956029 & -0.892176 & 0.000520 \\
\hline 9 & 7 & 0 & 2.286178 & 0.249186 & -0.001290 \\
\hline 10 & 13 & 0 & 1.639389 & 2.833332 & 0.001705 \\
\hline 11 & 6 & 0 & 0.963684 & -0.142517 & -0.001558 \\
\hline 12 & 1 & 0 & 4.039008 & -0.990962 & 0.001211 \\
\hline 13 & 1 & 0 & 2.408603 & -2.975379 & 0.002636 \\
\hline 14 & 7 & 0 & -2.642122 & -1.969061 & 0.001690 \\
\hline 15 & 1 & 0 & -2.705785 & -2.982296 & -0.001790 \\
\hline 16 & 1 & 0 & -3.490798 & -1.415688 & -0.006497 \\
\hline 17 & 1 & 0 & -2.277038 & 0.560250 & -0.000648 \\
\hline 18 & 7 & 0 & -3.684238 & 1.581266 & 0.001592 \\
\hline 19 & 1 & 0 & -4.300088 & 1.547350 & -0.818891 \\
\hline 20 & 1 & 0 & -4.297765 & 1.545963 & 0.823750 \\
\hline 21 & 1 & 0 & -3.272997 & 2.522522 & 0.001737 \\
\hline
\end{tabular}


CATION VI'

Standard orientation:

\begin{tabular}{|c|c|c|c|c|c|}
\hline Cente & Atomic & & tomic & Co & \\
\hline Number & Numbe & & Type & $Y$ & Z \\
\hline 1 & 8 & 0 & 1.497794 & -0.819707 & -0.076686 \\
\hline 2 & 6 & 0 & 0.384903 & -0.143248 & -0.014791 \\
\hline 3 & 7 & 0 & 0.392797 & 1.197206 & 0.036485 \\
\hline 4 & 13 & 0 & 3.040333 & $3-0.031863$ & -0.926675 \\
\hline 5 & 7 & 0 & 4.110388 & -0.743019 & 0.912682 \\
\hline 6 & 1 & 0 & 5.130054 & -0.772702 & 0.790858 \\
\hline 7 & 1 & 0 & 3.927422 & -0.127728 & 1.716752 \\
\hline 8 & 1 & 0 & 3.809479 & -1.685168 & 1.194623 \\
\hline 9 & 6 & 0 & -0.785954 & 1.888341 & 0.082409 \\
\hline 10 & 7 & 0 & -2.045938 & 1.368476 & 0.113590 \\
\hline 11 & 6 & 0 & -2.023874 & 0.047426 & 0.063466 \\
\hline 12 & 7 & 0 & -3.122364 & -0.821645 & 0.061341 \\
\hline 13 & 6 & 0 & -2.686041 & -2.099227 & -0.016169 \\
\hline 14 & 7 & 0 & -1.346839 & -2.088751 & -0.059566 \\
\hline 15 & 6 & 0 & -0.892083 & -0.776333 & -0.009865 \\
\hline 16 & 1 & 0 & -0.761121 & -2.919859 & -0.129359 \\
\hline 17 & 1 & 0 & -3.315096 & -2.982875 & -0.040433 \\
\hline 18 & 1 & 0 & -4.099972 & -0.534723 & 0.101454 \\
\hline 19 & 7 & 0 & -0.685160 & 3.233822 & 0.113341 \\
\hline 20 & 1 & 0 & -1.526776 & 3.800249 & 0.106774 \\
\hline 21 & 1 & 0 & 0.227437 & 3.670312 & 0.044188 \\
\hline
\end{tabular}

CATION VII'

Standard orientation:

\begin{tabular}{|c|c|c|c|c|c|}
\hline \multirow{2}{*}{$\begin{array}{l}\text { Center } \\
\text { Number }\end{array}$} & \multirow{2}{*}{$\begin{array}{l}\text { Atomic } \\
\text { Number }\end{array}$} & \multirow{2}{*}{\multicolumn{2}{|c|}{$\begin{array}{l}\text { Atomic } \\
\text { Type }\end{array}$}} & \multicolumn{2}{|c|}{ Coordinates (Angstroms) } \\
\hline & & & & $Y$ & 7 \\
\hline 1 & 8 & 0 & 2.723041 & 0.189100 & -0.000067 \\
\hline 2 & 6 & 0 & 1.479205 & 0.516342 & 0.003032 \\
\hline 3 & 7 & 0 & 1.062104 & 1.836622 & 0.000182 \\
\hline 4 & 6 & 0 & -0.288678 & 2.207800 & 0.002576 \\
\hline 5 & 7 & 0 & -1.290464 & 1.337472 & 0.006512 \\
\hline 6 & 7 & 0 & -4.434820 & -1.285038 & -0.001763 \\
\hline 7 & 1 & 0 & -4.843597 & -0.367236 & -0.211085 \\
\hline 8 & 1 & 0 & -4.818435 & -1.931896 & -0.699939 \\
\hline 9 & 1 & 0 & -4.828650 & -1.572425 & 0.901158 \\
\hline 10 & 6 & 0 & -0.896647 & 0.046239 & 0.000431 \\
\hline 11 & 7 & 0 & -1.648267 & -1.104943 & -0.000964 \\
\hline 12 & 6 & 0 & -0.753031 & -2.157296 & 0.002042 \\
\hline 13 & 7 & 0 & 0.519890 & -1.769926 & 0.005692 \\
\hline 14 & 13 & 0 & 3.132043 & -1.770823 & -0.003557 \\
\hline 15 & 6 & 0 & 0.429817 & -0.396647 & 0.004971 \\
\hline 16 & 1 & 0 & -1.084955 & -3.193100 & 0.001245 \\
\hline 17 & 1 & 0 & -2.728820 & -1.181645 & -0.001230 \\
\hline 18 & 7 & 0 & -0.554694 & 3.538673 & -0.046384 \\
\hline
\end{tabular}




$\begin{array}{rrrrrr}19 & 1 & 0 & -1.530496 & 3.803967 & 0.060546 \\ 20 & 1 & 0 & 0.140857 & 4.231336 & 0.213729 \\ 21 & 1 & 0 & 1.792969 & 2.550249 & -0.038881\end{array}$

